\title{
INFLUÊNCIA DOS HORMÔNIOS ESTERÓIODES SEXUAIS NO TEMPO DE ESQUELETIZAÇÃO DE RATOS WISTAR
}

\author{
INFLUENCE OF STEROID SEXUAL HORMONES ON SKELETIZATION TIME OF WISTAR RATS
}

Marco A. Guimarães', Ricardo M. Abrão², Andjara T.C. Soares³, Cláudia B. dos Santos ${ }^{4}$, José E. dos Santos ${ }^{5}$, Carmen C.S. Martin ${ }^{6}$

\begin{abstract}
'Docente, Centro de Medicina Legal (CEMEL), Departamento de Patologia, Faculdade de Medicina de Ribeirão Preto-USP (FMRP-USP). ${ }^{2}$ Pós-graduando, CEMEL, Departamento de Patologia, FMRP-USP, Docente da Universidade Paranaense (UNIPAR). ${ }^{3}$ Pós-graduanda, CEMEL, Departamento de Patologia, FMRP-USP. ${ }^{4}$ Docente, Departamento de Enfermagem Geral e Especializada, Escola de Enfermagem de Ribeirão Preto - USP. ${ }^{5}$ Docente, Departamento de Clínica Médica, FMRP-USP. ${ }^{6}$ Docente, CEMEL, Departamento de Patologia, FMRP-USP. Departamento de Medicina Legal, Ética Médica, Medicina Social e do Trabalho da Faculdade de Medicina - USP, São Paulo. Correspondência: Prof. Dr. Marco Aurelio Guimarães. Centro de Medicina Legal (CEMEL), Departamento de Patologia. Faculdade de Medicina de Ribeirão Preto - USP. Rua Tenente Catão Roxo no 2418 - CEP: 14051-140 - Ribeirão Preto - SP - Brazil.

Tel.: +55-16-36023358; fax: +55-16-36334476 / E-mail: mag@fmrp.usp.br
\end{abstract}

Guimarães MA, Abrão RM, Soares ATC, Santos CBS, Santos JE, Martin CCS. Influência dos hormônios esteróiodes sexuais no tempo de esqueletização de ratos wistar. Medicina (Ribeirão Preto) 2008; 41 (1): 35-42.

RESUMO: A estimativa do tempo de morte é um dos problemas mais complicados e difíceis da Medicina Legal devido aos diversos fatores que interferem nesse processo (temperatura, umidade, condições aeróbica e anaeróbica, presença de microrganismos e condições do solo). Fatores intrínsecos ao corpo freqüentemente não são relacionados à decomposição corporal. Para investigar se o processo de esqueletização sofre interferência dos hormônios esteróides sexuais foi realizado um trabalho experimental com ratos Wistar (30 machos e 60 fêmeas divididos em subgrupos em diferentes fases hormonais). Os animais foram cuidados até atingirem o peso entre 350 e $450 \mathrm{~g}$, quando foram mortos em câmara de $\mathrm{CO}_{2}$ e sepultados. A análise dos fatores ambientais (temperatura, umidade do ar e chuvas) e corporais (peso e gordura corporal) mostrou que a variação destes não interferiu no processo de esqueletização. Após as exumações, apenas o grupo de machos controles para testosterona apresentou esqueletização completa. Os machos castrados sem reposição de testosterona e os machos castrados com reposição de testosterona apresentaram esqueletização mínima. Todos os grupos das fêmeas apresentaram esqueletização parcial. A análise dos resíduos de putrefação dos grupos com esqueletização mínima e parcial evidenciou a presença de ácidos graxos característicos da composição de adipocera. Considerando-se que os dois grupos de animais (machos e fêmeas) foram sepultados no mesmo local, sob as mesmas condições ambientais e corporais, durante o mesmo intervalo de tempo, é possível concluir que os hormônios esteróides sexuais são responsáveis pela diferença observada na decomposição corpórea, sendo a testosterona o principal interveniente neste processo. Além disso, o modelo experimental também evidenciou o potencial de utilização para ser utilizado futuramente em estudos do processo de formação de adipocera.

Descritores: Decomposição corporal. Esqueletização. Esteróides sexuais. Adipocera. Rato. 


\section{1- INTRODUÇÃO}

A estimativa do tempo de morte é um dos problemas mais complicados e difíceis que se pode apresentar aos profissionais da Medicina Legal ${ }^{1,2,3}$. O Diagnóstico Cronológico da Morte, também conhecido como Tanatocronodiagnose ou Cronotanatognose, é a determinação do intervalo de tempo transcorrido entre o momento da morte e o encontro do cadáver. Quanto maior é esse intervalo, mais difícil será sua determinação, uma vez que o processo de decomposição do cadáver se constitui num processo e não num momento exato ${ }^{4,5}$.

Vários são os fatores internos e externos que influenciam a evolução das alterações relacionadas à morte ${ }^{6,7}$ e por isso sua cronologia varia de caso para caso $^{8}$. A putrefação não segue parâmetros rígidos em sua evolução, podendo ser rápida em certas condições e de uma lentidão surpreendente em outras ${ }^{5,9}$. Além disso, as transformações conservadoras dos cadáveres como a mumificação ou a saponificação podem modificar seus prazos de destruição, apesar de, em alguns casos, serem capazes de proporcionar, por si mesmas, indícios cronológicos ${ }^{10,11}$.

A saponificação é um processo transformativo conservador do cadáver, que consiste na mudança química da gordura corporal, através de hidrólise, em um composto ceroso similar aos sabões ${ }^{12,13}$. O processo de saponificação foi descrito em alguns cadáveres exumados no Cemitério dos Inocentes em Paris e, devido às características de uma substância que possuía propriedades intermediárias entre a gordura e a cera, foi denominada de adipocera ${ }^{9}$.

Para que haja a formação da adipocera é necessário que ocorram algumas condições ambientais e individuais específicas. Tal processo ocorre nas seguintes circunstâncias: 1) quando o cadáver está submergido em água parada ou de pouca corrente, 2) quando o cadáver está inumado em solo argiloso ou úmido ou 3) quando numerosos cadáveres estão enterrados uns em contato com os outros.

Algumas condições individuais também desempenham um papel importante na formação da adipocera. São elas: 1) idade: a adipocera é freqüente nas crianças, pois a quantidade de gordura subcutânea é proporcionalmente maior que nos adultos, 2) obesidade: é muito rara nos cadáveres de sujeitos magros e caquéticos, 3) condições patológicas: alcoolismo e outras intoxicações podem originar uma alteração gordurosa ou 4) sexo: o organismo feminino contém mais gordura do que o masculino ${ }^{4,9}$.
Considerando-se esta última condição, deve-se lembrar que tanto o esqueleto quanto a massa corporal magra e o conteúdo de gordura são iguais nos dois sexos durante a infância. Porém, após a puberdade, a massa corporal magra e o esqueleto crescem 1,5 vezes mais no homem, enquanto o conteúdo de gordura cresce duas vezes mais na mulher ${ }^{14}$. Contudo, não há na literatura uma descrição científica precisa sobre o papel do gênero masculino ou feminino na decomposição de cadáveres humanos, estando esta informação presente somente nos compêndios nacionais de Medicina Legal, como informação sem comprovação científica ${ }^{4}$.

Devido às dificuldades de se realizar estudos para isolar fatores específicos que interferem na decomposição do corpo humano ou de animais no meio ambiente, observações experimentais têm contribuído para o entendimento dos mecanismos biológicos envolvidos com os fenômenos de preservação de tecidos ${ }^{15 / 20}$.

Até hoje, a maioria dos estudos preocupa-se somente com fatores ambientais relacionados ao processo de decomposição corporal ${ }^{18 / 20}$, enquanto pouco se investiga sobre os fatores relacionados às características biológicas do corpo, tais como sexo, idade e proporção de gordura corporal. Além disso, há pouca ou nenhuma informação na literatura sobre o papel específico do gênero na decomposição de corpos. Um trabalho recente de nosso laboratório ${ }^{21}$ evidenciou experimentalmente que o processo de decomposição corporal é mais rápido em ratos machos do que em fêmeas quando sepultados exatamente sob as mesmas condições ambientais e exumados após 120 dias de sepultamento. Neste trabalho levantou-se a hipótese de que o perfil hormonal masculino com predomínio do hormônio esteróide sexual testosterona poderia ser responsável pela maior rapidez na decomposição corporal.

Sabendo-se que os hormônios sexuais são os responsáveis pela diferenciação e manutenção das características sexuais secundárias que se iniciam na puberdade $^{22,23}$, incluindo a modificação da proporção de gordura corporal observada no sexo feminino, cogitou-se que modificações do padrão hormonal em indivíduos do sexo masculino ou feminino poderiam levar a alterações no processo de decomposição corporal.

Tendo-se em vista a inviabilidade de investigação desta possibilidade em cadáveres humanos devido à complexidade que envolveria o estabelecimento do perfil hormonal no momento da morte, decidiu-se investigar a relação entre hormônios esteróides sexuais e a decomposição corporal, utilizando o mesmo 
modelo experimental com ratos Wistar machos e fêmeas previamente padronizado em nosso laboratório ${ }^{21}$.

O modelo experimental permite o estabelecimento de um perfil hormonal conhecido no momento da morte, de forma a possibilitar a análise de suas conseqüências no processo de decomposição corporal.

\section{2- MATERIAL E MÉTODOS}

\section{1- Aspectos Éticos da Pesquisa}

O trabalho foi submetido à revisão ética e aprovado pela Comissão de Ética no Uso de Animais (CEUA) do Campus de Ribeirão Preto da Universidade de São Paulo (USP), processo número 05.1.441.53.4.

\section{2- Animais}

Foram utilizados ratos, 30 machos e 60 fêmeas, da linhagem Wistar, com idade aproximada de 21 dias (desmamados). Os animais tiveram livre acesso à alimentação (ração comercial para roedores) e água, e foram mantidos sob estrito ciclo claro/escuro de 12/ $12 \mathrm{~h}$ (luzes tubulares fluorescentes acesas das $7 \mathrm{~h}$ às 19h) mantidas por controlador digital e temperatura de $23 \pm 2^{\circ} \mathrm{C}$.

O grupo dos machos foi dividido em três subgrupos: 10 machos castrados sem reposição de testosterona (MCST), 10 machos castrados com reposição de testosterona (MCCT) e 10 machos controles para testosterona (MCoT). O grupo das fêmeas foi dividido em seis subgrupos: 10 fêmeas controles na fase proestro $(\mathrm{FCoP}), 10$ fêmeas controles na fase estro (FCoE), 10 fêmeas controles na fase diestro (FCoD), 10 fêmeas castradas sem reposição de hormônio (FCSH), 10 fêmeas castradas com reposição de estrógeno (FCCE) e 10 fêmeas castradas com reposição de progesterona (FCCP).

\section{3- Ciclo Estral}

As fêmeas que apresentaram ciclo estral irregular após avaliação diária durante 21 dias foram selecionadas para ooforectomia bilateral (castração) e composição dos grupos castrado com ou sem reposição hormonal. As demais, com ciclo estral regular, formaram os grupos controles em diferentes fases do ciclo estral, avaliado pela última vez instantes antes da morte. Assim, foram formados os subgrupos de acordo com a fase do ciclo em que se encontravam.

\section{4- Cirurgia}

Dos 30 ratos machos utilizados, 20 foram submetidos à cirurgia para retirada dos testículos entre o $21^{\circ}$ e o $25^{\circ}$ dia de vida. Das 60 fêmeas, 30 foram selecionadas conforme descrito anteriormente e submetidas a ooforectomia bilateral com aproximadamente 50 dias de vida. Para as cirurgias, os animais foram anestesiados com tribromoetanol (Acros Oragnics ${ }^{\circledR}$, New Jersey, USA) a 2,5\% diluído em solução salina $0,9 \%$, sendo injetado $1 \mathrm{~mL}$ de solução para cada $100 \mathrm{~g}$ de peso corpóreo por via intra-peritonial.

\section{5- Reposição hormonal}

O subgrupo MCCT, duas semanas após a cirurgia, com aproximadamente trinta dias de vida, recebeu a primeira dose de reposição hormonal e após 21 dias o subgrupo recebeu a segunda e última dose de hormônio. A reposição hormonal foi feita utilizando Durateston ${ }^{\circledR}$ (decanoato de testosterona, fenilpropianato de testosterona, isocaproato de testosterona, propionato de testosterona) com concentração de $0,6 \mu \mathrm{g} / \mathrm{mL}$ de óleo para injeção subcutânea, sendo $0,1 \mathrm{~mL}$ de solução para cada $100 \mathrm{~g}$ de peso corpóreo por via intra-muscular.

Para os subgrupos FCCE e FCCP, a reposição hormonal foi realizada por quatro dias consecutivos quando os animais estavam com aproximadamente 150 dias de vida e pesando entre $350-450 \mathrm{~g}$. O subgrupo FCCE recebeu doses de cipionato de estradiol (17 $\beta$ cipionato de estradiol - Sigma ${ }^{\circledR}$, St. Louis, MO, USA) de $0,1 \mu \mathrm{g} / \mathrm{g}$ p.c., ou seja, $10 \mu \mathrm{g} / 100 \mu \mathrm{l}$ de veículo oleoso para cada $100 \mathrm{~g}$ de peso corpóreo, por via subcutânea e o subgrupo FCCP recebeu doses de progesterona (Sigma ${ }^{\circledR}$, St. Louis, MO, USA) de $2,5 \mu \mathrm{g} / \mathrm{g}$ p.c., ou seja, $250 \mu \mathrm{g} / 100 \mu \mathrm{l}$ de veículo oleoso para cada $100 \mathrm{~g}$ de peso corpóreo, por via subcutânea.

\section{6- Morte e Sepultamento}

Machos e fêmeas foram observados até atingirem o peso entre 350 e $450 \mathrm{~g}$, com controle de peso corporal em momentos específicos, até serem mortos em câmara de $\mathrm{CO}_{2}{ }^{24}$. Confirmada a morte dos animais, eles foram envolvidos individualmente em gaze e algodão e colocados em caixas de madeira, simulando uma urna, medindo $47 \times 23 \times 9 \mathrm{~cm}$, divididas em cinco compartimentos de $20,2 \times 8 \times 7,8 \mathrm{~cm}$, para conter um animal em cada, sendo então fechadas com tampa de madeira. $\mathrm{O}$ fundo de cada compartimento foi forrado com uma camada de folha de alumínio e 
uma camada de papel filtro sobre esta, para evitar o contato direto dos corpos com a madeira.

As urnas foram identificadas e colocadas numa caixa de cimento $(65 \times 49$ x $44 \mathrm{~cm})$ enterrada no chão para servir de sepultura. Dentro dessa sepultura as urnas foram colocadas sobre tijolos mantendo uma distância de $18 \mathrm{~cm}$ do fundo, para evitar seu contato com líquidos que pudessem se acumular no fundo, evitando exposição diferenciada à água. Cada subgrupo foi dividido em duas urnas, sendo que uma foi colocada em contato com tijolo e a outra sobre a primeira.

A sepultura foi tampada para evitar o acesso de predadores. Desse modo, todos os animais foram mantidos sob as mesmas condições ambientais. Após 120 dias de sepultamento, a exumação dos animais foi realizada.

Os restos mortais foram levados ao Laboratório de Antropologia Forense do Centro de Medicina Legal (CEMEL) da Faculdade de Medicina de Ribeirão Preto -USP para realização das análises e registro fotográfico. Ao término das análises o material restante foi eliminado como lixo hospitalar, segundo a normatização vigente ${ }^{24}$.

\section{7- Temperatura, umidade relativa do ar e chuvas}

O experimento procedeu-se sob as condições ambientais do local. Em intervalos de 24 horas foram feito registros das temperaturas máxima e mínima, da umidade relativa do ar e da ocorrência ou não de chuva, com as finalidade de obter um registro da variáveis ambientais externas à sepultura.

\section{8- Pesos}

O peso dos animais foi registrado em quatro momentos: (a) inicial: quando os animais foram recebidos no biotério; (b) ante-mortem: durante o intervalo antes e após a administração dos hormônios; (c) no momento da morte e (d) no momento da exumação.

\section{8- Gordura}

No momento da morte dos animais foi feita a medida de gordura no dorso de cada um utilizando-se um paquímetro.

\section{8- Análises Estatísticas}

Os resultados foram organizados em uma base de dados utilizando o software SPSS $®$ (Statistical Package for Social Sciences), versão 10.1 para o
Windows ${ }^{\circledR}$ e os dados tratados, estatisticamente, através da análise descritiva e inferencial. O nível de significância utilizado foi $\alpha=0,05$. Foram feitas as seguintes análises situações: (a) Temperatura e Umidade Relativa do Ar - Teste t de Student; (b) Normalidade dos valores na distribuição dos pesos e na medida de gordura no dorso do animal - KolmogorovSmirnov; (c) Pesos e medida de gordura entre os nove subgrupos - teste da Análise de Variância (ANOVA) com o teste Post Hoc de Tukey e (d) Peso antemortem: análise realizada somente para os subgrupos FCSH, FCCE e FCCP através do teste paramétrico t-pareado que compara duas amostras dependentes. Todas as médias foram apresentadas com seu desvio padrão (DP).

\section{9- Análise Cromatográfica}

Para a pesquisa de ácidos graxos foram colhidas amostras da massa cadavérica proveniente dos restos da decomposição corpórea. A extração dos ácidos graxos foi feita a $80^{\circ} \mathrm{C}$ com clorofórmio-metanol ${ }^{25}$. Para a identificação do composto, foi utilizado o cromatógrafo gás-líquido HP5890 Série II utilizando a coluna Techonology IMC cat. 19091N-213 da Agilent. A pressão de nitrogênio e hidrogênio foi mantida a $30 \mathrm{~mL} / \mathrm{min}$ e a pressão do ar sintético a $300 \mathrm{~mL} / \mathrm{min}$. Foi feita análise programada com temperatura inicial da coluna a $180^{\circ} \mathrm{C}$ e com temperatura final a $250^{\circ} \mathrm{C}$. A temperatura de injeção era de $220^{\circ} \mathrm{C}$ e a do detector era de $260^{\circ} \mathrm{C}$.

\subsection{0- Graus de esqueletização}

Foi criado um padrão para descrever a decomposição corpórea tendo como referência o próprio esqueleto do animal, chamado de grau de esqueletização. Apenas três graus foram estabelecidos para a esqueletização: a completa, a parcial e a mínima. Também foram descritas a presença de pele ou pêlos.

A esqueletização foi considerada: (a) completa - quando o esqueleto era visível, com os ossos soltos, secos e sem resíduos, sem aderência de ligamentos, tendões e restos musculares e completa ausência de tecidos moles; (b) parcial - quando partes do esqueleto eram visíveis, apresentando restos musculares ou aderência de ligamentos, tendões e massa cadavérica recobrindo algumas partes do esqueleto; (c) mínima quando os corpos estavam completamente recobertos pela massa cadavérica ou até com presença de órgãos e vísceras, apresentando muita umidade, impossibilitando a visualização do esqueleto. 


\section{3- RESULTADOS}

\section{1- Temperatura, umidade relativa do ar e chuvas}

Para o grupo dos machos foi registrada temperatura máxima de $42^{\circ} \mathrm{C}$ e mínima de $5^{\circ} \mathrm{C}$, obtendo-se uma média geral de $25,2 \pm 11,1^{\circ} \mathrm{C}$. Para as fêmeas a temperatura máxima foi $46^{\circ} \mathrm{C}$ e a mínima $13^{\circ} \mathrm{C}$, com média geral de $29,6 \pm 11,6^{\circ} \mathrm{C}$. Na comparação entre as médias gerais das temperaturas dos dois grupos foi observada diferença estatisticamente significativa $(p=0,014)$.

A média da umidade relativa do ar para o grupo dos machos e o das fêmeas, foi, respectivamente, $88,7 \pm 7,0 \%$ e $87,7 \pm 7,8 \%$. Não foi observada diferença estatisticamente significativa entre as médias $(p=0,240)$.

Em relação às chuvas, não foi observado o acúmulo de água dentro da sepultura.

\section{2- Normalidade dos Valores}

O teste de Kolmogorov-Smirnov mostrou que houve normalidade na distribuição dos pesos inicial, ante-mortem, no momento da morte e na exumação, assim como na medida de gordura no dorso do animal.

\section{3- Pesos}

Analisando as médias dos pesos dos diferentes subgrupos considerados, o teste ANOVA mostrou não haver diferenças estatisticamente significativas para os pesos inicial $(p=0,085)$ e no momento da morte $(p=0,445)$. No momento da exumação, houve diferenças estatisticamente significativa $(p<0,0001)$ entre o peso dos animais. O teste de Tukey mostrou a seguinte relação entre os grupos:

$\mathrm{MCCT}>\mathrm{MCST}>\mathrm{FCoP}=\mathrm{FCoE}>\mathrm{MCOT}>\mathrm{FCoD}=\mathrm{FCSH}=\mathrm{FCCP}=\mathrm{FCCE}$

Quando foram comparados os pesos antes e após a administração dos hormônios, o teste t pareado mostrou diferenças estatisticamente significativa para os subgrupos FCCE $(p=0,045)$ e FCCP $(p=0,0001)$. O teste também foi realizado para o subgrupo $\mathrm{FCSH}$, no mesmo intervalo de tempo, e mostrou diferença estatisticamente significativa $(p=0,0004)$.

Analisando as médias das medidas de gordura nos diferentes subgrupos, o teste ANOVA mostrou haver diferenças estatisticamente significativas $(p=0,016)$. O teste de Tukey mostrou que apenas dois grupos diferiram entre si e esta diferença, em relação às médias das medidas de gordura, se apresentou da seguinte maneira: FCoE < FCCE.

\section{4- Exumação dos Animais}

Após 120 dias de inumação, as urnas foram retiradas da sepultura e todas elas se apresentavam íntegras, com o mesmo aspecto externo. No interior das caixas havia a presença de fauna cadavérica recobrindo o algodão que envolvia os corpos. Esse algodão foi cortado com tesoura para evitar mudanças na posição dos restos dos corpos. Não foi observado contato dessa fauna com os corpos dos animais.

\section{5- Graus de Esqueletização}

O subgrupo MCoT foi o único que apresentou um padrão de esqueletização completa (Figura 1A). Os subgrupos FCCP, FCoE, FCoD, FCoP, FCSH e FCCE apresentaram-se com um padrão de esqueletização parcial (Figura 1B). Os subgrupos MCST e MCCT apresentaram um padrão de esqueletização mínima (Figura 1C).

\section{6- Massa Cadavérica}

Todas as massas cadavéricas analisadas por cromatografia gasosa revelaram a presença dos ácidos palmítico, esteárico, oléico e linolêico em sua composição. Esses resultados confirmaram que a massa cadavérica tratava ser adipocera.

\section{4- DISCUSSÃO}

Com o intuito de verificar isoladamente o efeito dos hormônios esteróides sexuais na decomposição corporal de ratos Wistar, outras variáveis relacionadas às condições ambientais (temperatura, umidade, chuva e local de sepultamento) foram controladas.

Contudo, observou-se diferença significativa $(p=0,014)$ entre a temperatura a qual o grupo dos machos ficou exposto $\left(25,2 \pm 11,1^{\circ} \mathrm{C}\right)$ e a temperatura do grupo das fêmeas $\left(29,6 \pm 11,6^{\circ} \mathrm{C}\right)$. A temperatura média para as fêmeas foi maior em $4,4^{\circ} \mathrm{C}$. Mesmo assim, nenhum subgrupo apresentou o padrão de esqueletização completa como aconteceu com o subgrupo MCoT que ficou exposto a temperatura menor. Sabese que no tempo frio a decomposição corpórea é mais lenta ou, até mesmo, inibida, e é mais difícil se estimar o tempo de morte de um corpo na época do ano em que a temperatura oscila entre morna e fria $^{16}$. Assim, era de se esperar que o grupo das fêmeas sofresse uma decomposição mais acelerada em função da temperatura mais alta, mas isto não ocorreu sugerindo que o efeito do tratamento hormonal ou do sexo predominam sobre a temperatura. 

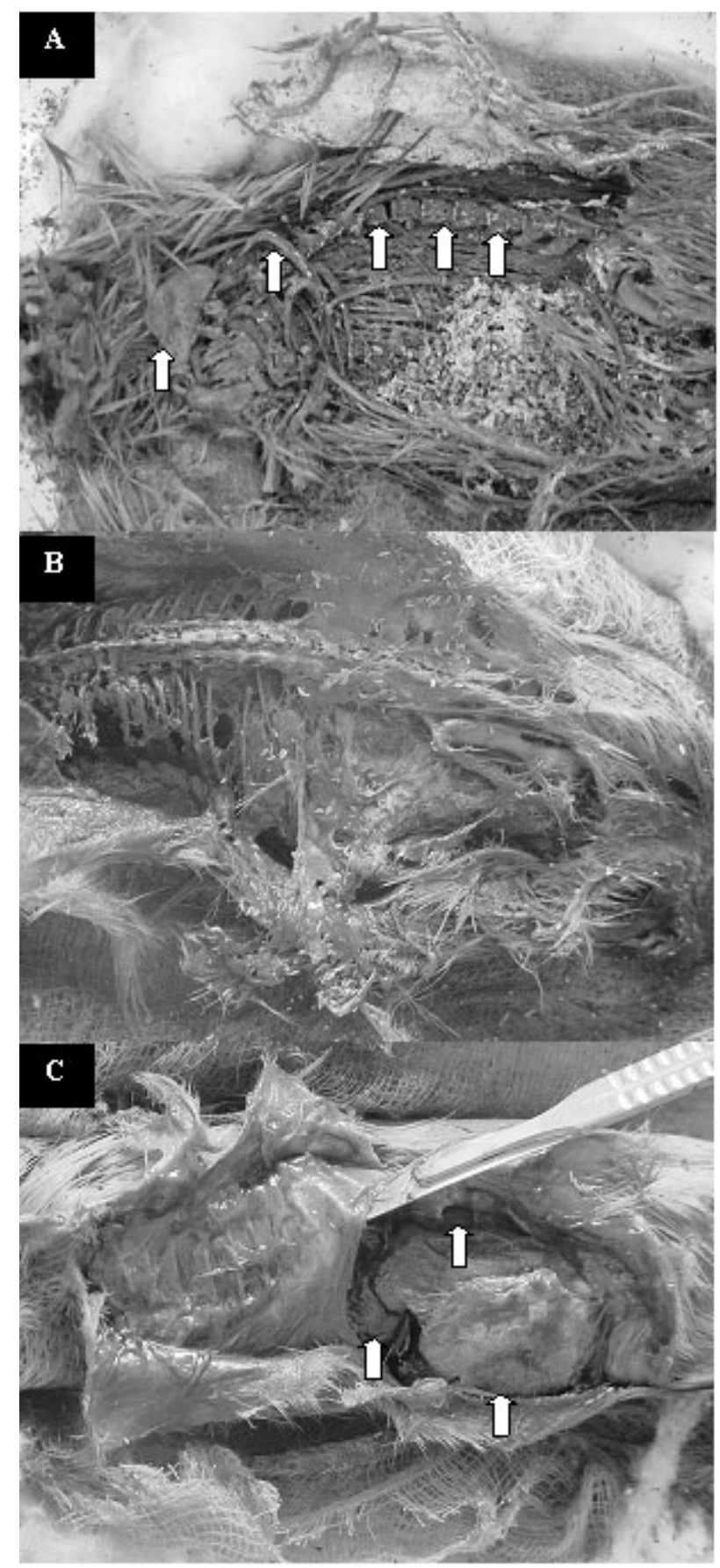

Figura 1: Animais após a exumação. (A) Esqueletização completa: presença de pele, esqueleto visível, ossos soltos (setas indicam escápula, costelas e vértebras), secos e sem resíduos, sem aderência de ligamentos, tendões e restos musculares e completa ausência de tecidos moles. (B) Esqueletização parcial: partes do esqueleto visível (setas), mas com presença de restos musculares, aderência de ligamentos, tendões e massa cadavérica recobrindo algumas partes do esqueleto. (C) Esqueletização mínima: esqueleto não visível, corpo recoberto pela massa cadavérica com presença de órgãos e vísceras (seta).
Com relação ao peso corporal, foi confirmado pelo teste ANOVA que não houve diferenças estatisticamente significativas entre os pesos iniciais $(p=0,085)$ e os pesos no momento da morte $(p=0,445)$. Assim, pode-se afirmar que todos os animais foram inumados com peso corporal estatisticamente semelhante, o que neutraliza sua influência nos resultados obtidos.

Comparando-se os pesos antes e após a administração dos hormônios, o teste de t pareado mostrou diferenças estatisticamente significativas para os subgrupos FCCE $(p=0,045)$ e FCCP $(p=0,0001)$, que receberam reposição hormonal por quatro dias consecutivos antes de serem mortos. Para constatarmos se essa diferença estava associada à administração do hormônio, o subgrupo FCSH foi comparado durante o mesmo intervalo de tempo. Também foi observada diferença estatisticamente significativa $(p=0,0001)$, o que indica que a variação de peso encontrada não foi devido à aplicação dos hormônios.

$\mathrm{Na}$ exumação dos animais, tanto para os machos quanto para as fêmeas, não foi observado acúmulo de líquidos no interior da sepultura. A água ambiental pode ter uma grande influência na formação da adipocera, embora em sua ausência o processo também seja possível. Neste caso, a água necessária para a formação da adipocera, ao menos em uma primeira fase, provém dos tecidos corporais que se desidratam sensivelmente após a morte ${ }^{9}$.

A decomposição corpórea se apresentou de maneira variada. $\mathrm{O}$ grupo dos machos estava dividido em três padrões hormonais distintos. O padrão estabelecido para o subgrupo MCoT foi a esqueletização completa. Foi o único subgrupo entre machos e fêmeas que apresentou esse padrão. Os subgrupos MCST e MCCT apresentaram entre si o mesmo padrão de esqueletização mínima. As comparações feitas entre os subgrupos dos machos mostraram grande diferença no processo de decomposição corpórea. Enquanto o subgrupo MCoT apresentou a maioria dos animais com o esqueleto visível, com ossos soltos, secos e sem resíduos, para as mesmas condições ambientais e o mesmo intervalo de tempo, os subgrupos MCST e MCCT apresentaram animais que continham órgãos e vísceras bastante preservados.

O grupo das fêmeas estava divido em seis fases hormonais. Comparando-se o grupo dos machos com o grupo das fêmeas não houve semelhança entre os padrões apresentados, pois todos os subgrupos das fêmeas apresentaram grau de esqueletização parcial, o que sugere que o padrão hormonal de predomínio de testosterona nos machos influencie o processo de decomposição corporal acelerando-o. 
Em mamíferos, as fêmeas têm uma porcentagem maior de gordura corporal em relação aos ma$\operatorname{chos}^{23}$. Essa característica está associada ao padrão hormonal feminino, ou seja, concentrações de estrógeno e progesterona mais altas e concentrações de andrógenos mais baixas. Além disso, concentrações mais altas de andrógenos estão associadas com porcentagem menor de gordura corporal ${ }^{26,27}$.

A literatura considera que maiores porcentagens de gordura corporal estão associadas com maior probabilidade de formação de adipocera durante o processo de esqueletização ${ }^{4,28}$. $\mathrm{O}$ mecanismo de formação da adipocera tem sido extensamente estudado, mas ainda não está totalmente elucidado ${ }^{16,17}$.

A formação da adipocera inibe a putrefação devido à acidez crescente dos tecidos e à desidratação causada pelo consumo de água na hidrólise, retardando o crescimento e a propagação das bactérias $^{29}$. Todavia, há casos em que a adipocera compromete apenas a superfície do cadáver, preservando integralmente os conjuntos musculares, permitindo assim um estudo médico-legal mais consistente ${ }^{4}$.

A constatação dos diferentes tipos de ácidos graxos (ácidos palmítico, esteárico, oléico e linolêico) encontrados nas massas cadavéricas detectados como característicos da composição da adipocera constitui um achado de importância no modelo experimental utilizado, uma vez que o mesmo pode ser reproduzido em futuros estudos do processo de formação desta substância, sabidamente um evento difícil de ser obtido em condições experimentais ${ }^{12,15-20}$.

Assim, além da detecção do efeito de hormônios esteróides sexuais, principalmente a testosterona, na decomposição corporal, o modelo em ratos Wistar permitiu o estabelecimento de uma alternativa para o estudo da formação de adipocera.

\section{6 - CONCLUSÕES}

As análises realizadas indicaram que a variação dos fatores ambientais (temperatura, umidade do ar e chuvas) e corporais (peso e gordura corporal) não interferiram no processo de decomposição.

Portanto, pode-se concluir que os hormônios esteróides sexuais são responsáveis pelas diferenças observadas na decomposição corporal. Além disso, a testosterona apresentou papel de destaque em relação à progesterona e ao estrógeno no processo de esqueletização, sobressaindo até mesmo em relação à ausência da testosterona endógena (animais não castrados).

$\mathrm{E}$, finalmente, a detecção de adipocera nos grupos experimentais com decomposição parcial ou mínima torna-se uma alternativa viável para futuros estudos do processo de formação desta substância relacionada ao processo de saponificação.

Guimarães MA, Abrão RM, Soares ATC, Santos CBS, Santos JE, Martin CCS. Influence of steroid sexual hormones on skeletization time of Wistar rats. Medicina (Ribeirão Preto) 2008; 41 (1): 35-42.

ABSTRACT: The establishment of the postmortem interval is one of the most complicated and difficult tasks in forensic investigation because of many factors that affect this process (temperature, humidity, aerobic and anaerobic conditions, presence of microorganisms and soil conditions). Body intrinsic factors frequently not are related to de body decomposition. To investigate if the skeletonization process is under interference of sexual steroid hormones an experimental investigation with Wistar rats was done (30 male and 60 females divided in sub-groups at different hormonal phases). The animals were raised until they reach body weight between 350 and $450 \mathrm{~g}$, when were killed in a $\mathrm{CO}_{2}$ chamber and buried. Analysis of the environmental (temperature, humidity and rain) and corporal factors (body weight and body fat) showed that variation in these factors did not interfere on the skeletonization process. After the exhumation, only the male control group for testosterone showed complete skeletonization. The castrated males with no testosterone reposition and the castrated males with testosterone reposition showed minimum skeletonization. All female groups showed partial skeletonization. Analysis of the putrefaction residues from the groups with partial and minimum skeletonization showed the presence of the characteristic fatty acids of adipocere composition. Considering that both animal groups (males and females) were buried at the same place, under the same environmental and body conditions, during the same time interval, it is possible to conclude that sexual steroid hormones are responsible for the observed difference in body decomposition, being testosterone the main intervenient factor in this process. Besides, the experimental model also evidenced its potential use for future studies in the process of adipocere formation.

Keywords: Body decomposition. Skeletization. Sexual steroids. Adipocere. Rat. 


\section{7- REFERÊNCIAS}

1 - Goff ML, Flynn MM. Determination of postmortem interval by arthropod succession: a case study from the Hawaiian islands. J Forensic Sci 1991; 36: 607-14.

2 - Grellner W, Glenewinkel F. Exhumations: synopsis of morphological and toxicological findings in relation to the postmortem interval. Survey on a 20-year period and review of the literature. Forensic Sci Int 1997; 90: 139-59.

3 - Hércules HC. Cronologia da morte. In: Hércules HC, atualizador. Medicina legal. $32^{\mathrm{a}}$ ed. Rio de Janeiro: Freitas Bastos; 1997. p. 140-74.

4 - França GV. Medicina legal. 6‥ ed. Rio de Janeiro: GuanabaraKoogan; 2001.

5 - Cañadas EV. Data de la muerte y outros problemas tanatológicos médico-legales. In: Cañadas EV, editor. Medicina legal y toxicologia. $6^{\underline{a}}$ ed. Barcelona: Masson.; 2005. p. 242-52.

6 - Campobasso CP, Di Vella G, Introna F. Factors affecting decomposition and Diptera colonization. Forensic Sci Int 2001; 120: 18-27.

7 - Rothschild MA, Schmidt V, Schneider V. Adipocere: problems in estimating the length of time since death. Med Law 1996; 15: $329-35$.

8 - Schoenly K, Griest K, Rhine S. An experimental field protocol for investigating the postmortem interval using multidisciplinary indicators. J Forensic Sci 1991; 36: 1395415.

9 - Gonzalo JC. Procesos conservadores del cadáver. In: Cañadas EV, editor. Medicina legal y toxicologia. $6^{\underline{a}}$ ed. Barcelona: Masson; 2005. p. 214-24.

10 - Galloway A. The process of decomposition: a model from the Arizona-Sonoran desert. In: Haglund WD, Sorg MH, editors. Forensic taphonomy: the postmortem fate of human remains. $1^{\text {st }}$ ed. Boca Raton, Fla: CRC Press; 1997. p. 13950 .

11 - Haglund WD. Disappearance of soft tissue and the disarticulation of human remains from aqueous environments. J Forensic Sci 1993; 38: 806-15.

12 - Mann RW, Bass WM, Meadows L. Time since death and decomposition of the human body: variables and observations in case and experimental field studies. $J$ Forensic Sci 1990; 35: 103-11.

13 - Forbes SL, Stuart BH, Dadour IR, Dent BB. A preliminary investigation of the stages of adipocere formation. J Forensic Sci 2004; 49: 566-74.

14 - Douglas CR. Fisiologia da gônada masculina. In: Douglas CR, editor. Tratado de Fisiologia aplicada à saúde. $5^{\mathrm{a}}$ ed. São Paulo: Robe Editorial; 2002. p. 1303-12.

15 - Forbes SL, Stuart BH, Dent BB, Fenwick-Mulcahy S. Characterization of adipocere formation in animal species. $J$ Forensic Sci 2005; 50: 633-40.
16 - Takatori T. Investigations on the mechanism of adipocere formation and its relation to other biochemical reactions. Forensic Sci Int 1996; 80: 49-61.

17 - Takatori T. The mechanism of human adipocere formation. Leg Med 2001; 3: 193-204.

18 - Forbes SL, Dent BB, Stuart BH. The effect of soil type on adipocere formation. Forensic Sci Int 2005; 154: 35-43.

19 - Forbes SL, Stuart BH, Dent BB. The effect of the method of burial on adipocere formation. Forensic Sci Int 2005; 154 : 44-52.

20 - Forbes SL, Stuart BH, Dent BB. The effect of the burial environment on adipocere formation. Forensic Sci Int 2005; 154: $24-34$

21 - Rocha FS, Guimarães MA, Martin CCS. Tempo do processo de esqueletização de ratos Wistar em diferentes tipos de sepultamento [resumos]. XV Reunião Anual da Federação de Sociedade de Biologia Experimental (FESBE), Caxambu MG; 2000. p. 234.

22 - Fodor M, Van Leeuwen FW, Swaab DF. Differences in postmortem stability of sex steroid receptor immunoreactivity in rat brain. J Histochem Cytochem 2002; 50: 641-9.

23 - Trudeau F, Shephard RJ, Arsenault F, Laurencelle L. Changes in adiposity and body mass index from late childhood to adult life in the Trois-Rivieres study. Am J Hum Biol 2001; 13: 349-55.

24 - Andersen ML, D'Almeida V, Ko GM, Kawakami R, Martins PJF, Magalhães LE, Tufik S. Princípios éticos e práticos do uso de animais de experimentação. São Paulo: Universidade Federal de São Paulo (UNIFESP), 2004.

25 - Folch J, Lees M, Stanley GHS. A simple method for the isolation and purification of total lipids from animal tissues. $J$ Biol Chem 1956; 226: 497-509.

26 - Münzer T, Harman SM, Hees P, Shapiro E, Christmas C, Bellantoni MF, Stevens TE, O'Connor KG, Pabst KM, St Clair $\mathrm{C}$, Sorkin JD, Blackman MR. Effects of GH and/or sex steroid administration on abdominal subcutaneous and visceral fat in healthy aged women and men. J Clin Endocrinol Metab 2001; 86: 3604-10.

27 - Vermeulen A, Goemaere S, Kaufman JM. Testosterone, body composition and aging. J Endocrinol Invest 1999; 22: 110-6.

28 - Knight B. Pathophysiology of death - Postmortem decomposition. In: Knight B, editor. Forensic Pathology. London: Edward Arnold; 1991. p. 58-65.

29 - Pfeiffer S, Milne S, Stevenson RM. The natural decomposition of adipocere. J Forensic Sci 1998; 43: 368-70.

Recebido para publicação em 27/08/2007

Aprovado para publicação em 13/05/2008 